\title{
REESTRUTURADO EMPANADO ELABORADO COM RESÍDUO DE PESCADA AMARELA (Cynoscion acoupa) E ADIÇÃO DA FIBRA DE MARACUJÁ
}

\author{
E. Moura ${ }^{1}$, E. A. F. Araújo ${ }^{1}$; L. F. H. Lourenço ${ }^{1}$, C. L. Sousa ${ }^{1}$, M. R. S. Peixoto Joele ${ }^{2}$ \\ ${ }^{1}$ Universidade Federal de Pará, Programa de Pós-graduação em Ciência e Tecnologia de Alimentos \\ ${ }^{2}$ Instituto Federal de Educação, Ciência e Tecnologia do Pará. Castanhal (PA) \\ E-mail para contato: luciahl@ufpa.br
}

\begin{abstract}
RESUMO - O objetivo deste trabalho foi elaborar um produto reestruturado empanado a partir do resíduo da pescada amarela (Cynoscion acoupa) e enriquecido com fibras obtidas da casca do maracujá. Os resíduos foram submetidos à análises físicas, físicoquímicas e microbiológicas. A massa base do reestruturado foi elaborada a partir de um planejamento fatorial completo $2^{2}$, totalizando 11 formulações, para selecionar a melhor concentração de farinha da casca do maracujá e fécula de mandioca através de avaliação sensorial. Após a escolha da melhor formulação, foi estudado o melhor tempo de fritura (1,30 minutos, 2,30 min, 3,30 min). O produto selecionado foi com a adição de $1,47 \%$ de farinha da casca do maracujá, $5 \%$ de fécula de mandioca e tempo de fritura de 3 minutos e 30 segundos, obtendo-se um produto de alto valor nutricional e boa aceitação.
\end{abstract}

\section{INTRODUÇÃO}

Os resíduos da industrialização do pescado representam um sério problema para a planta industrial, principalmente por serem poluentes e de difícil descarte, interferindo na eficiência do processo produtivo. A importância nutricional do pescado como alimento humano e a questão ambiental, são razões que apontam para a necessidade de utilizar a totalidade do pescado capturado. Além disso, seu aproveitamento integral tende a contribuir para o aumento do consumo de proteína animal, pois diversas tecnologias têm surgido com possíveis utilizações dos resíduos como fontes alimentares e com boa aceitabilidade (Stori et al., 2002).

A pescada amarela (Cynoscion acoupa) um dos mais importantes gêneros de sciaenídeos em termos comerciais, ganhando destaque entre os peixes com maior volume de processamento nos estados do Pará e Amazonas. Em relação ao beneficiamento, o desperdício, considerando cabeça e resíduos, representa mais de $60 \%$ de sua produção (Boscolo, 2001).

O aproveitamento de fontes subutilizadas de pescado, tais como, desperdícios do processamento ou mesmo espécies que tradicionalmente não são comercializadas, constitui uma preciosa fonte de proteína animal. Contudo, estas fontes de proteína não são muitas vezes utilizadas pelo fato de não serem sensorialmente atrativas e se degradarem com elevada facilidade. $O$ desenvolvimento de 
produtos de pescado reestruturados constitui uma saída para estas matérias-primas subvalorizadas (Borderias, 2005).

Os produtos da pesca têm um elevado valor nutricional e apresentam propriedades funcionais, no entanto não contêm fibra. A adição de fibras dietéticas a produtos de pescado reestruturados é de grande interesse, não apenas pelo papel funcional que estas têm a nível tecnológico, mas também, pelo fato de permitirem desenvolver produtos alimentares funcionais com diversos benefícios para a saúde (Sánchez-Alonso e Borderías, 2007).

O objetivo deste trabalho foi desenvolver um reestruturado empanado com resíduo de pescada amarela e enriquecido com fibras alimentares.

\section{MATERIAL E MÉTODOS}

\subsection{Matérias-primas}

As matérias-primas utilizadas para obter o produto reestruturado foram: surimi de resíduos da filetagem de pescada amarela (Cynoscion acoupa), farinha da casca do maracujá. Todos os resíduos foram doados congelados $\left(-20^{\circ} \mathrm{C}\right)$ por indústrias localizadas na região nordeste do Estado do Pará. Os outros ingredientes e aditivos foram adquiridos no comércio local.

\subsection{Surimi do resíduo de pescada amarela}

O resíduo de pescada amarela foi descongelado, lavado com água clorada a $5 \mathrm{mg} / \mathrm{L}$ por 10 minutos e separado manualmente as espinhas e escamas. O processo de obtenção do surimi foi baseado em metodologia proposta por Ogawa e Maia (1999). O resíduo resultante da separação manual foi triturado em multiprocessador doméstico à baixa temperatura para evitar a desnaturação das proteínas. $\mathrm{O}$ material triturado foi lavado, por quatro vezes, com água e gelo (mantendo à $5^{\circ} \mathrm{C}$ ), na proporção de 1:4 (peixe:água). Em seguida, feita a prensagem, adicionados os crioprotetores sorbitol $(3 \%)$ e tripolifosfato de sódio $(0,3 \%)$ para a obtenção do surimi que foi embalado à vácuo e congelado à $-20^{\circ} \mathrm{C}$, para posterior utilização.

\subsection{Farinha da casca de maracujá}

A farinha de maracujá foi elaborada segundo metodologia proposta por Pena et al. (2008). A casca de maracujá foi cortada em cubos de $1 \mathrm{~cm}^{3}$ e a secagem foi realizada em estufa (modelo BIPOLAR, marca SOC. FABBE LTDA) com circulação e renovação de ar.

A farinha de maracujá foi obtida na melhor condição de temperatura até valor máximo de umidade de $8,0 \%$, que é estabelecido para a farinha de soja desengordurada (ANVISA, 1978). Esse valor foi utilizado em virtude de não haver legislação específica para farinha de casca de maracujá. $\mathrm{O}$ material desidratado foi triturado para obtenção de granulometria homogênea e embalado à vácuo.

\subsection{Delineamento experimental para estabelecer a formulação do reestruturado}


Após testes preliminares foi definida a formulação base do reestruturado: surimi (97,8\%), condimento completo ARISCO® $(2,0 \%)$ e glutamato monossódico $(0,2 \%)$. A partir desta formulação foi realizado um delineamento composto central rotacional $2^{2}$ (Tabela 1), para determinar os percentuais de farinha da casca de maracujá e fécula de mandioca a serem adicionadas à massa.

No delineamento foram avaliadas a influência das variáveis independentes (\% de farinha da casca de maracujá e \% de fécula de mandioca) sobre as respostas sensoriais: sabor amargo (influência da fibra da farinha da casca do maracujá), textura (macia, porém consistente) e aceitação global.

Tabela 1 - Matriz codificada do delineamento experimental $2^{2}$ com os respectivos valores reais das variáveis estudadas.

\begin{tabular}{|c|c|c|c|c|}
\hline \multirow{2}{*}{ Ensaios } & \multicolumn{2}{|c|}{ Variáveis codificadas } & \multicolumn{2}{c|}{ Variáveis reais } \\
\cline { 2 - 5 } & $\begin{array}{c}\text { Farinha maracujá } \\
(\%)\end{array}$ & $\begin{array}{c}\text { Fécula mandioca } \\
(\%)\end{array}$ & $\begin{array}{c}\text { Farinha maracujá } \\
(\%)\end{array}$ & $\begin{array}{c}\text { Fécula mandioca } \\
(\%)\end{array}$ \\
\hline 01 & -1 & -1 & 2,50 & 2,50 \\
\hline 02 & +1 & -1 & 7,50 & 2,50 \\
\hline 03 & -1 & +1 & 2,50 & 7,50 \\
\hline 04 & +1 & +1 & 7,50 & 7,50 \\
\hline 05 & $-1,41$ & 0 & 1,47 & 5,00 \\
\hline 06 & $+1,41$ & 0 & 8,52 & 5,00 \\
\hline 07 & 0 & $-1,41$ & 5,00 & 1,47 \\
\hline 08 & 0 & $+1,41$ & 5,00 & 8,52 \\
\hline 09 & 0 & 0 & 5,00 & 5,00 \\
\hline 10 & 0 & 0 & 5,00 & 5,00 \\
\hline 11 & 0 & 0 & 5,00 & 5,00 \\
\hline
\end{tabular}

As formulações foram moldadas à uma temperatura de $\pm 1^{\circ} \mathrm{C}$, devido a maior facilidade de corte. Em seguida, os produtos foram pré-fritos à temperatura de $190^{\circ} \mathrm{C}$ por 45 segundos, envolvidos em filme plástico e armazenados congelados a $-20^{\circ} \mathrm{C}$, até a realização da análise sensorial.

Os reestruturados foram pré-fritos a temperatura média de $195^{\circ} \mathrm{C}$ durante 1 ' $30^{\prime}$ e avaliados sensorialmente por 35 provadores não treinados, de ambos os sexos. As amostras foram servidas de forma monádica e avaliadas quanto aos atributos sabor e textura utilizando escala não estruturada de 9 cm e aceitação global utilizando escala hedônica (Dutcosky, 2011). O reestruturado com melhor pontuação nos atributos avaliados foi submetido a processo de empanamento.

\subsection{Determinação do tempo de fritura do reestruturado empanado}

O reestruturado recebeu um sistema de cobertura de acordo com metodologia descrita por Bonacina e Queiroz (2007), o predust foi feito com farinha de trigo, o batter foi realizado com clara de ovo e o breading com farinha industrializada para empanar. Em seguida, foram estabelecidos através de testes preliminares os tempos de fritura (1,30 min; 2,30 min e 3,30 min) a $195^{\circ} \mathrm{C}$. 


\section{9 a 22 de outubro de 2014 \\ Florianópolis/SC}

Os reestruturados fritos foram avaliados sensorialmente por 35 provadores não treinados, de ambos os sexos, servidos de forma monádica. Foi utilizada escala estruturada para avaliar os atributos aparência, cor, brilho, uniformidade, firmeza, sabor amargo e sabor a peixe e escala hedônica para o teste de aceitação global. Para os atributos sabor amargo e sabor peixe, buscou-se a nota mínima.

\subsection{Análises microbiológicas}

Foram realizadas as análises de Salmonella sp., Estafilococos coagulase positiva e coliformes a $45^{\circ} \mathrm{C}$ na farinha da casca de maracujá, no resíduo de pescada amarela, no surimi e no reestruturado. Análises de Bacillus cereus e psicrófilos foram feitas somente na farinha e no reestruturado, respectivamente e realizadas de acordo com Brasil (2001a). As análises seguiram metodologia do Compendium of Methods for the Microbiological Examination of Foods (Downes \& Ito, 2001).

\subsection{Análises físicas e físico-químicas}

$\mathrm{Na}$ farinha da casca de maracujá, no resíduo de pescada amarela, no surimi e no reestruturado foi feita a composição centesimal (AOAC, 1997) e a atividade de água $\left(\mathrm{a}_{\mathrm{w}}\right)$ com o Termohigrômetro digital AQUAlab 3TE da Decagon, a $25^{\circ} \mathrm{C} \pm 0,5$. A fibra alimentar total foi obtida pelo método enzimático gravimétrico de acordo com 985.29 e 991.43 da AOAC (1997). Bases voláteis totais (BVT) no resíduo e surimi de pescada amarela segundo Brasil (1981).

\subsection{Análise estatística}

Os resultados do delineamento experimental e análise sensorial foram avaliados pela análise de variância (ANOVA) e as médias pelo teste de Tukey $(\mathrm{P}<0,05)$ com o programa o Statistica 5.0.

\section{RESULTADOS E DISCUSSÃO}

\subsection{Caracterização microbiológica do resíduo e surimi da pescada amarela}

Nas amostras analisadas do resíduo e do surimi da pescada amarela não foram detectadas presença de Salmonella spp. As contagens de estafilococos coagulase positiva $\left(<1 \times 10^{1} \mathrm{UFC} / \mathrm{g}\right) \mathrm{e}$ coliformes a $45^{\circ} \mathrm{C}(<3,0 \mathrm{NMP} / \mathrm{g})$ encontravam-se dentro dos padrões estabelecidos pela legislação brasileira (Brasil, 2001a). Estes resultados demonstram que o resíduo e o surimi encontram-se em condições higiênico-sanitárias adequada para a elaboração do produto reestruturado.

\subsection{Caracterização física e físico-química do resíduo e surimi de pescada amarela}

Os resultados das análises físicas e físico-químicas encontram-se na Tabela 2. O elevado teor de umidade, Aw e pH do resíduo e surimi de pescada amarela indicam alta perecibilidade, sendo necessária a utilização de métodos de conservação eficientes que controlem as possíveis alterações, principalmente as de origem microbiológica (Rebouças et al., 2012). O valor de N-BVT indica que o resíduo encontrava-se em excelente estado de frescor (Brasil, 1999). 
Tabela 2 - Média dos resultados da composição física e físico-química do resíduo e surimi de pescada amarela

\begin{tabular}{|l|c|c|}
\hline Determinação & Resíduo & Surimi \\
\hline${\text { Umidade }(\%)^{1}}^{1}$ & $80,43 \pm 0,25$ & $82,12 \pm 0,09$ \\
\hline${\text { Lipídios totais }(\%)^{1}}^{1}$ & $2,20 \pm 0,02$ & $2,04 \pm 0,11$ \\
\hline Proteínas $(\%)^{1}$ & $17,53 \pm 0,20$ & $14,59 \pm 0,01$ \\
\hline Cinzas $(\%)^{1}$ & $0,36 \pm 0,01$ & $0,33 \pm 0,01$ \\
\hline Bases voláteis totais $(\mathrm{mgN} / 100 \mathrm{~g})$ & $8,40 \pm 0,42$ & $6,65 \pm 0,24$ \\
\hline pH & $7,00 \pm 0,04$ & $6,97 \pm 0,03$ \\
\hline Aw & $0,98 \pm 0,00$ & $0,98 \pm 0,00$ \\
\hline
\end{tabular}

${ }^{1}$ Base úmida

O resíduo e o surimi de pescada amarela são classificados como matéria-prima de baixo teor de gordura devido ao valor de lipídios encontrado (Pigott e Tucker, 1990). O teor de proteínas obtido é similar ao valor encontrado por Bonancina e Queiroz (2007). A diminuição no teor de proteínas do surimi em relação ao resíduo ocorreu devido às sucessivas lavagens, que eliminam as proteínas sarcoplasmáticas solúveis em água.

\subsection{Caracterização microbiológica, física e físico-química da farinha da casca de maracujá}

Os resultados de Coliformes a $45^{\circ} \mathrm{C}$, Bacillus cereus e Salmonella sp da farinha da casca de maracujá estão de acordo com os padrões estabelecidos (Brasil, 2001a).

A umidade da farinha $(5,30 \%)$ é considerada baixa, pois de acordo com os padrões regulamentados pela legislação vigente para farinhas em geral, este valor deve ser menor que 14,0\% (ANVISA, 1978), o que favorece o aumento da vida de prateleira. O teor proteico da farinha da casca de maracujá $(4,96 \%)$ varia de acordo com o processo de obtenção ou ainda por diferentes locais de plantio e época da colheita (Pena et al., 2008). Os resultados da análise de fibras $(42,00 \%)$ indicam que este subproduto apresenta alto teor de fibras totais solúveis e insolúveis, podendo ser boa alternativa como enriquecimento nutricional.

\subsection{Elaboração do reestruturado empanado}

A Tabela 3 apresenta as médias dos atributos avaliados nas formulações estabelecidas pelo planejamento experimental, onde a formulação $5(1,47 \%$ de farinha de maracujá e $5 \%$ de fécula de mandioca), obteve maior média para a aceitação global $(84,1 \%)$. Observa-se que a adição de até $5 \%$ de farinha de casca de maracujá nos reestruturados obteve bons resultados e a formulação 5 que obteve melhor avaliado em todos atributos.

Tabela 3 - Médias atribuídas pelos provadores aos atributos sensoriais

\begin{tabular}{|l|l|l|l|}
\hline Formulação & Aceitação global & Sabor & Textura \\
\hline
\end{tabular}




\begin{tabular}{|l|c|c|c|}
\hline 01 & $7,05^{\mathrm{ac}}$ & $6,85^{\mathrm{ad}}$ & $6,62^{\mathrm{ac}}$ \\
\hline 02 & $4,34^{\mathrm{b}}$ & $4,24^{\mathrm{b}}$ & $3,51^{\mathrm{e}}$ \\
\hline 03 & $6,85^{\mathrm{ac}}$ & $6,38^{\mathrm{ad}}$ & $5,75^{\mathrm{cd}}$ \\
\hline 04 & $4,88^{\mathrm{bd}}$ & $4,90^{\mathrm{bc}}$ & $4,30 \mathrm{be}$ \\
\hline 05 & $7,57^{\mathrm{a}}$ & $7,68^{\mathrm{a}}$ & $7,14^{\mathrm{a}}$ \\
\hline 06 & $5,88^{\mathrm{cd}}$ & $5,55^{\mathrm{bd}}$ & $5,91^{\mathrm{ac}}$ \\
\hline 07 & $5,88^{\mathrm{cd}}$ & $5,92^{\mathrm{cde}}$ & $5,34^{\mathrm{bc}}$ \\
\hline 08 & $6,97^{\mathrm{ac}}$ & $6,81^{\mathrm{ad}}$ & $6,72^{\mathrm{ad}}$ \\
\hline 09 & $7,11^{\mathrm{a}}$ & $6,98^{\mathrm{ae}}$ & $6,78^{\mathrm{ad}}$ \\
\hline 10 & $6,97^{\mathrm{ac}}$ & $6,75^{\mathrm{ad}}$ & $6,35^{\mathrm{ac}}$ \\
\hline 11 & $6,57^{\mathrm{ac}}$ & $6,41^{\mathrm{ad}}$ & $6,08^{\mathrm{ac}}$ \\
\hline
\end{tabular}

Médias seguidas de mesma letra na coluna não diferem significativamente entre si $(\mathrm{p}<0,05)$.

Observa-se que a adição de até $5 \%$ de farinha de casca de maracujá nos reestruturados obteve bons resultados e a formulação 5 foi melhor avaliada em todos atributos. Com esta formulação foi realizado o estudo do melhor tempo de fritura (Tabela 4).

Tabela 4 - Resultado das médias atribuídas pelos provadores aos atributos analisados

\begin{tabular}{|l|c|c|c|}
\hline Atributos & 1 min e $30 \mathrm{seg}$ & $2 \min$ e $30 \mathrm{seg}$ & $3 \mathrm{~min} \mathrm{e} 30 \mathrm{seg}$ \\
\hline Aparência & $5,11^{\mathrm{a}}$ & $6,71^{\mathrm{a}}$ & $6,91^{\mathrm{a}}$ \\
\hline Cor & $4,11^{\mathrm{a}}$ & $5,11^{\mathrm{a}}$ & $5,37^{\mathrm{a}}$ \\
\hline Brilho & $4,2^{\mathrm{a}}$ & $5,31^{\mathrm{b}}$ & $5,65^{\mathrm{ab}}$ \\
\hline Uniforme & $6,08^{\mathrm{a}}$ & $6,17^{\mathrm{a}}$ & $6,42^{\mathrm{a}}$ \\
\hline Firmeza & $6,22^{\mathrm{a}}$ & $6,22^{\mathrm{a}}$ & $6,27^{\mathrm{a}}$ \\
\hline Sabor Amargo & $3,31^{\mathrm{a}}$ & $2,40^{\mathrm{a}}$ & $3,65^{\mathrm{a}}$ \\
\hline Sabor Peixe & $5,62^{\mathrm{a}}$ & $5,97^{\mathrm{a}}$ & $5,80^{\mathrm{a}}$ \\
\hline Ac. Global & $5,88^{\mathrm{a}}$ & $6,17^{\mathrm{a}}$ & $6,88^{\mathrm{ab}}$ \\
\hline
\end{tabular}

Médias seguidas de mesma letra na linha não diferem significativamente entre si $(\mathrm{p}<0,05)$.

A amostra com tempo de fritura de 3 minutos e 30 segundos obteve maiores médias nos atributos aparência, cor, uniformidade, firmeza, sabor amargo e sabor de peixe, apresentando diferença significativa $(\mathrm{p} \leq 0,05)$ apenas para os atributos brilho e aceitação global.

\subsection{Análises microbiológicas, físicas e físico-químicas do reestruturado}

Os resultados de Salmonella sp, Estafilococos Coagulase + , Coliformes a $45^{\circ} \mathrm{C}, e$ Psicrófilos do reestruturado estão de acordo com os padrões estabelecidos pela RDC n 12, (Brasil, 2001a).

O percentual de proteínas $(15,61 \%)$ obtido está de acordo com a legislação vigente, a qual recomenda o mínimo de $10 \%$ de proteína (Brasil, 2001). A umidade e os teores de proteínas e cinzas encontrados no reestruturado empanado apresentam variações em relação a Bonacina e Queiroz, 
(2007) que podem ser explicadas pelas diferenças no processo de obtenção ou ainda pela utilização de diferentes componentes no empanamento.

A quantidade de fibras no reestruturado empanado pré-frito foi de 0,56 $\pm 0,01$, ressaltando-se que a farinha de casca de maracujá foi a principal responsável por pela inclusão do teor de fibras, por se tratar de um produto com "alto teor de fibras" (42g/100g). Luvielmo e Dill (2008) ao adicionar 2\% de metilcelulose na farinha de cobertura de produtos fritos empanados obtiveram até $10,51 \%$ de redução no teor de gordura, indicando que a inclusão de fibras diminui a absorção de óleo durante o processo.

A inclusão de fibras em produtos de origem animal aumenta o valor nutricional destes produtos auxiliando no consumo diário recomendado. A American Dietetic Association (ADA) recomenda, para adultos sadios, a ingestão de 20 a 35g de fibras/dia ou 10 a $13 \mathrm{~g}$ de fibras para cada $1.000 \mathrm{Kcal}$ ingeridas. Desta forma, a ingestão do produto elaborado seria responsável por 2,8\% do total diário recomendado para um adulto sadio.

\section{CONCLUSÕES}

O reestruturado empanado com adição de $1,47 \%$ de farinha da casca do maracujá e $5 \%$ de fécula de mandioca e tempo de fritura de 3 minutos e 30 segundos foi selecionado como o melhor produto de acordo com os atributos analisados. A inclusão de fibras em produtos de origem animal aumenta o valor nutricional auxiliando no consumo diário recomendado. Agregar valor a resíduos agroindustriais é de interesse ambiental, econômico, científico e tecnológico.

\section{AGRADECIMENTOS}

\section{Ao CNPq pela bolsa de Mestrado}

\section{REFERENCES}

ANVISA. AGÊNCIA NACIONAL DE VIGILÂNCIA SANITÁRIA - Resolução - CNNPA nº12, de 1978 http://www.anvisa.gov.br/legis/resol/12_78_farinhas.htm; Acesso em: 30 jun/2009.

AOAC. Association Analitical Chemists. Official methods of analysis of the Association Analitical Chemists, 16th ed, v. 1, p. 1002 - 1020, Washington, DC. 1997.

BONACINA, M; QUEIROZ, M. I. Elaboração de empanado a partir da corvina (Micropogonias furnieri). Ciênc. Tecnol. Aliment., v. 27, p. 544-552. 2007.

BORDERÍAS, A. J., Y PÉREZ-MATEOS, M. Sucedáneos de productos pesqueros: tecnología de reestructuración. Alim. Nutr. Salud, v. 12, p. 61-70. 2005.

BOSCOLO, W.R. Desempenho e características de carcaça de machos revertidos de tilápias do Nilo (Oreochromis niloticus), linhagens tailandesa e comum nas fases iniciais e de crescimento. Rev. Bras. Zootec., v. 30, p.1391-1396, 2001. 
SÁNCHEZ-ALONSO, I.; BORDERÍAS, A.J. Technological effect of red grape antioxidant dietary fibre added to minced fish muscle. I. J. Food Sci.Tech., v. 43, p. 1009-1018, 2008.

BRASIL. Ministério da Saúde, Agência Nacional de Vigilância Sanitária - ANVISA. Resolução RDC $\mathrm{n}^{\circ} 12$ de 02 de janeiro de 2001. Aprova Regulamento Técnico sobre os padrões microbiológicos para alimentos. Diário Oficial. Brasília, DF. 10 de janeiro de 2001a.

BRASIL. Instrução Normativa $\mathrm{n}^{\circ}$ 06, de 06/02/2001. Regulamento Técnico de Identidade e Qualidade de Empanados. Ministério da Agricultura, Pecuária e Abastecimento, Brasília 2001b.

BRASIL. Ministério da Agricultura, Pecuária e Abastecimento. Instrução Normativa ${ }^{\circ}$ 20, de 21 de julho de 1999. Oficializa os métodos analíticos físico-químicos para controle de produtos cárneos e seus ingredientes/sal e salmoura. Diário Oficial da União, Brasília, DF. 1999.

BRASIL. Ministério da Agricultura. Laboratório Nacional de Referência Animal. Métodos Analíticos Oficiais para Controle de Produtos de Origem Animal e seus Ingredientes. Brasília, DF: Ministério da Agricultura, v. 2, cap. 11. Pescado Fresco. 1981.

DUTCOSKY, S.D. Análise sensorial de alimentos. $3^{\mathrm{a}}$ ed. Paraná:Champagnat. 426 p. 2011.

DOWNES, F. P.; ITO, K. (Eds.). Compendium of methods for the microbiological. Examinations of Foods (4th. ed.). Washington, DC: APHA. 2001.

SÁNCHEZ-ALONSO, I.; BORDERÍAS, A.J. Technological effect of red grape antioxidant dietary fibre added to minced fish muscle. I. J. Food Sci.Tech., v. 43, p. 1009-1018, 2008.

LUVIELMO, M. M, de; DILL, D. D. Utilização da goma metilcelulose para redução da absorção de gordura em produtos empanados. Semina: Ciên. Ex. Ter., v. 29, p. 107-118, 2008.

PENA, R. S.; SILVA, D. M. S.; MENDONÇA N. B.; ALMEIDA, M. D. C. Estudo da secagem da fibra residual do maracujá. Rev. Bras. Tecnol. Agroind., v. 2, p.1-13, 2008.

PIGOTT, G; TUCKER, B. Sea food effects of technology on nutrition, 1st edit, Edit Marcel Dekker, INC, New York, USA, 1990.

STORI, F. T., BONILHA, L. E. C., PESSATTI, M. L. Proposta de aproveitamento dos resíduos das indústrias de beneficiamento de pescado de Santa Catarina com base num sistema gerencial de bolsa de resíduos. In: Social, Inst. Ethos de Empresas e Responsabilidade Econômica, responsabilidade social das empresas. São Paulo, 2002.

REBOUÇAS, M.C.; RODRIGUES, M.C.P.; CASTRO, R.J.S.; VIEIRA, J.M.M. Caracterização do concentrado protéico de peixe obtido a partir dos resíduos da filetagem de tilápia do Nilo. Semina: Ciênc. Agrár., v. 33, p. 697-704, 2012. 